\title{
THEORETICAL ANALYSIS OF IRON-BORE SUPERCONDUCTING UNDULATORS
}

\section{Ben-Zvi, R. Fernow, J. Gallardo, G. Ingold, W. Sampson, M. Woodle} Brookhaven National Laboratory, Upton, NY $11973^{\text {: }}$ Tel 516-282-3523; Fax 516-282-3000

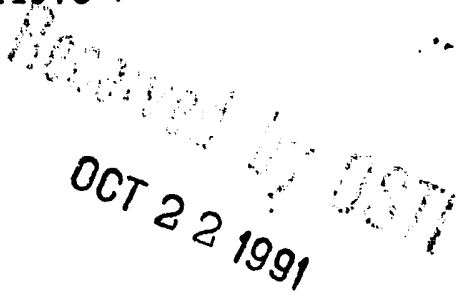

\begin{abstract}
The unavoidable undulator field errors due to machining tolerances of the yokes lead to a reduction of the gain in comparison with the case computed with an ideally perfect undulator. We describe a mathematical model of errors in iron-dominated micro-undulators introduced by manufacturing tolerances. Explicit expressions for the perturbation fields are derived. We use these fields to calculate the first, second and phase integrals describing the effects on the electron trajectory. We conclude that the intrinsic tolerances achievable with wire electric discharge machining do not significantly affect the performance of the Accelerator Test Facility free-electron laser.

\section{DISCLAIMER}

This report was prepared as an account of work sponsored by an agency of the United States Government. Neither the United States Government nor any agency thereof, nor any of their employees, makes any warranty, express or implied, or assumes any legal liability or responsibility for the accuracy, completeness, or usefulness of any information. apparat us, product, or process disclosed, or represents that its use would not infringe privately owned rights. Reference herein to any specific commercial product, process, or service by trade name, trademark, manufacturer, or otherwise does not necessarily constitute or imply its endorsement, recommendation, or favoring by the United States Government or any agency thereof. The views and opinions of authors expressed herein do not necessarily state or ref. $\rightarrow t$ those of the United States Government or any agency thereof.
\end{abstract}

This manuscript bas been authored under contract number DE-AC02-76CH00016 with the U.S. Department of Energy. Accordingly, the U.S. Government retains a non-exclusive, royalty-free license to publish or reproduce the published form of this contribution, or allow others to do so, for U.S. Government purposes. 


\section{Introduction}

A visible free-electron laser (FEL) is presently under development at the Accelerator Test Facility (ATF) at the Brookhaven National Laboratory. The present status of the experiment as well as an overview of the facility is described in another article [1] submitted to this conference.

The novel undulator used is a very compact super-ferric sub-centimeter period $\left(\lambda_{w}=\right.$ $0.88 \mathrm{~cm}$ ) device with a total length $L_{w}=60 \mathrm{~cm}$ and a gap $g=0.44 \mathrm{~cm}$. The advantages of a superconducting iron core undulator have been stressed in several publications [2], [3]; here we only mention that the required high precision machining of the yokes can be obtained rather inexpensively using the wire electric discharge method (WEDM). The details of the design as well as the performance of several prototype pieces are described in an accompanying article [4]

In this work, we describe a mathematical model developed to account for machining errors in the poles and notches milled in the low carbon steel yokes that made the undulator. To be able to identify the effect of different sources of wiggler errors on the gain of the FEL and consequently, its performance and final power, we simplify the analysis by neglecting errors in the period $\lambda_{w}$ and the saturation effects of the iron (the permeability is assumed to be infinite $\mu=\infty$ ).

We discuss two different types of uncorrelated random machining errors denoted by type $a$ and $b$ and a third systematic error due to assembly tolerances as type $c$. Type $a$ (gap error) arises from a perturbation of the pole facing the midplane of the device with the consequence of a local change of the nominal wiggler gap $(g=0.44 \mathrm{~cm})$, see Fig. 1a).

Type $b$ (pole length error) arises from a shaving of the pole thickness perpendicular to the midplane, see Fig. 1b). Finally, type $c$ (alignment error) arises from a relative longitudinal shift of the two yokes of the micro-undulator, see Fig. 1c).

The explicit expressions for the error fields are used in the third section to realistically model a random distribution of gap and pole-thickness errors. No ad-hoc assumptions regarding the field behavior underneath the pole or the functional form of the correlation function between errors is made. A numerical simulation of the electron trajectories provide limits on the machining tolerances of the undulator yoke by imposing constrains on the 
first, second and phase integrals of the $B_{y}$ component of the undulator field. To satisfy these conditions the rms wiggler exror must be less than $\frac{\delta B}{B} \lesssim 0.4 \%$. $\delta B$ represents the standard deviation of random mechanical errors and its equivalent for the corresponding magnetic field errors.

\section{Mathematical Model}

To simplify the treatment we assume a two dimensional planar geometry $(y, z)$ for the undulator. This is a good approximation for the BNL micro-wiggler [5](see Fig. 1) and will hold approximately correct for horizontal pole sizes larger than the wiggler gap.

We solve the Laplace equation for the magnetic scalar potential $\Phi(y, z)$ in the region between the planes $y= \pm \frac{d}{2}$, determined by the surface of the poles in the upper and lower yokes of the undulator [6]. Due to the periodicity in the z-direction, we choose $\Phi(y, z)=$ $\Phi(y,-z)$. The assumption of $\mu=\infty$ leads to boundary conditions $\Phi\left( \pm \frac{d}{2}, z_{\text {pole }}\right)= \pm B_{S}$, (i.e. constant magnetic field of alternating sign), at the poles of both jaws; between them there are no physical constraints other than the continuity of $\Phi\left( \pm \frac{d}{2}, z\right)$. The different choices of the interpolating function between poles have no effect on the fundamental of the field and it will only affect the harmonic content of the fields. For the super-ferric micro-undulator under construction at BNL the simplest and physically correct assumption is to take the boundary conditions as a linear function of $z$ between the poles and constant at the pole; this yields a harmonic falls off $\sim \frac{a_{1}}{n(n-1)}$.

A general solution of the Laplace equation is $\Phi^{( \pm)}(y, z)=\sum_{n=1}^{\infty} A_{n} \sinh \frac{\pi n}{l}\left(y \pm \frac{d}{2}\right) \sin \frac{\pi \pi}{l} z$ where $\ell=\frac{1}{2} \lambda_{w}$ is haif the wiggler wavelength, $A_{n}$ are constants to be determined imposing the appropriate boundary conditions and $\Phi^{( \pm)}(y, z)$ is the poiential function in the region under consideration with the boundary condition at the upper jaw given by $\pm B_{S}$ and $\Phi^{(+)}\left(-\frac{d}{2}, z\right)=0$. Similarly for $\Phi^{(-)}(y, z)$ with $\mp B_{S}$ in the lower jaw of the wiggler.

The magnetic fields on axis read,

$$
B_{y}(0, z)=\sum_{n=1,3,5 \ldots}^{\infty} B_{n}^{\circ} \frac{\cosh \frac{\pi n}{l} \frac{1}{2} d}{\cosh \frac{\pi \pi}{l} d} \sin \frac{\pi n}{\ell} z \quad \text { and } \quad B_{z}(0, z)=0
$$

the complete expressions for the magnetic fields are given in Appendix A of Ref. 5 . 


\section{Error Fields}

The mathematical model discussed previously adapts well to the inclusion of wiggler errors due to macnining tolerances. We proceed first to ccnsider a single pole error in the upper jaw. We derive the fields induced by such perturbation by using the superposition principle and a number of practical prescriptions by $K$. Halbach $[7,8]$ that describe the removal or addition of a thin sheet of iron to the poles pieces.

\section{rap error fields}

The effects of a gap error at a single pole in the upper jaw (see Fig. 1a), considered to be a perturbation of the boundary shape of the problem discussed in the previous section, is computed following the prescription depicted graphically in Fig. 2, i.e. the field between the two planes is the sum of the field for a perfect wiggler plus the perturbation field produced by a localized strip in the upper plane $\left(y=\frac{1}{2} d\right)$ at potential $\Delta \Phi$.

The model for the perturbation term consists of two parallel planes at $y= \pm \frac{d}{2}$, both at scalar potential $\Phi(y, z)=0$ except on a localized strip on the upper plane at the position $z_{\text {pole }}$ of the gap error where the boundary condition is $\Delta \Phi_{S}$. To calculate $\Delta \Phi_{S}$, we follow $K$. Halbach's suggestion that the addition or extraction of a thin sheet of iron of width $\delta$ at the pole tooth of the upper jaw introduces a dipole layer of magnitude $D=\delta \frac{1}{4 \pi} B_{S}\left(z_{\text {pole }}\right)$ and the discontinuity of the potential is $\Delta \Phi_{S}=4 \pi D$. In this problem, there is no periodicity in $z$ and therefore we change the Fourier sum in the usual solution into a Fourier integral. The standard limit procedure yields the Green function, $G(y z \mid \zeta)=$ $\frac{1}{2 x} \int_{-\infty}^{\infty} d k \frac{\sinh k\left(\frac{1}{d} d+y\right)}{\sinh k d} \cos k(\zeta-z)$ and the scalar potential in the region between both planes is given by $\phi(y, z)=\int_{-\frac{1}{2} h}^{+\frac{1}{2} h} d \zeta \Delta \Phi_{S}(\zeta) G(y z \mid \zeta)$ where $h$ is the extent of a single pole and $\Delta \Phi_{S}(\zeta)$ is the potential at the boundary surface representing the gap error. For $-\frac{1}{2} h<$ $z<+\frac{1}{2} h$ we obtain $\phi(y, z)=2 \frac{\Delta \Phi_{S}}{d} y+\frac{\Delta \Phi_{S}}{\pi} \sum_{n=1}^{\infty}\left(\frac{1}{n}\right) \sin \frac{n \pi}{d}\left(y+\frac{d}{2}\right)\left[X^{n}+Y^{n}\right]$ where we have used $X=\exp \left[-\frac{\pi}{d}\left(z+\frac{1}{2} h\right)\right]$ and $Y=\exp \left[\frac{\pi}{d}\left(z-\frac{1}{2} h\right)\right]$. The field errors on axis are,

$$
b_{y}(0, z)=-\frac{\Delta \Phi_{S}}{d}\left[\frac{1}{1+X^{2}}+\frac{1}{1+Y^{2}}\right] \quad, \quad b_{z}(0, z)=\frac{\Delta \Phi_{S}}{d}\left[\frac{X}{1+X^{2}}-\frac{Y}{1+Y^{2}}\right]
$$

At any other position $z$ away from the pole $\left(|z|>\frac{1}{2} h\right)$,

$$
\phi(y, z)=-\frac{\Delta \Phi_{S}}{\pi} \sum_{n=1}^{\infty}\left(\frac{1}{n}\right) \sin \frac{n \pi}{d}\left(y+\frac{d}{2}\right)\left[-T^{n}+S^{n}\right]
$$


in this case the error fields on axis are,

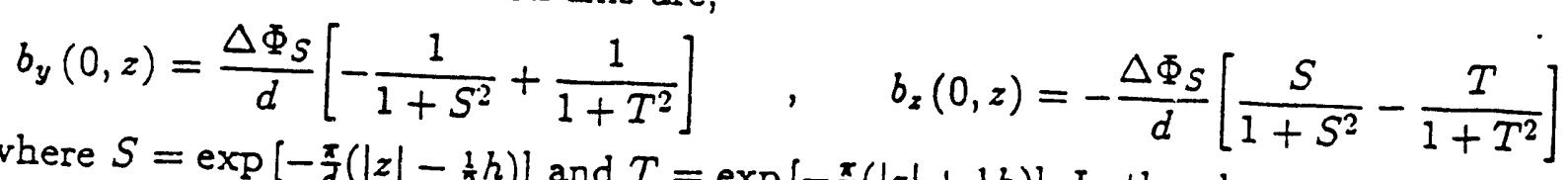

where $S=\exp \left[-\frac{\pi}{d}\left(|z|-\frac{1}{2} h\right)\right]$ and $T=\exp \left[-\frac{\pi}{d}\left(|z|+\frac{1}{2} h\right)\right]$. In the above equations $z$ is the relative longitudinal position respect to the pole $z_{\text {pole }}$.

By examination of the perturbation fields it is noted that below the pole where the error is located the field is not $2 \frac{\Delta \phi}{d}$ as expected if the error has no influence on the remaining poles in the upper jaw. The correction teims represent the interaction of the single localized error with the rest of the wiggler poles. Furthermore, the actual transverse field sampled by the electrons is a function of the relative position with respect the center of the pole, i.e. $\frac{\Delta \Phi_{S}}{d}\left(\frac{1}{1+e^{-\frac{2 \pi}{d} h}}+\frac{1}{2}\right) \leq b_{y}(0, z) \leq 2 \frac{\Delta \Phi_{S}}{d} \frac{1}{1+e^{-\frac{z}{d} h}}$. Also, far from the pole with the gap error, the perturbation field shows a fast decay as we move away from it.

These results allow us to make a more realistic modelling of a random distribution of gap errors in an iron-dominated wiggler, in particular regarding the axial dependence of the error fields over the extension of the pole and their correlation properties.

\section{Pole length error field}

We follow a similar argument as in the previous section. The effect of perturbation of the width of the pole tooth in the upper jaw of the wiggler is depicted graphically in Fig. 3

The error field is in this case the solution of the Laplace equation between two planes at $y= \pm \frac{d}{2}$ with boundary conditions $\frac{\partial \Phi\left( \pm \frac{d}{2}, z\right)}{\partial y}=0$ everywhere, except in the strip at the pole position $z_{\text {pole }}$ in the upper jaw where the boundary condition is $-\frac{\partial \Phi}{\partial y}=\frac{\delta}{h} B_{S}\left(z_{\text {pole }}\right) \equiv N_{S}$; here $\delta$ is the change of the tooth longitudinal dimension, $h$ is the extent of the pole and $B_{S}\left(z_{\text {pole }}\right)$ is the vertical magnetic field at the pole surface of a perfect wiggler. This can be shown to follow from flux conservation, $(h \pm \delta) B_{y}^{\prime}=h B_{y}$ and consequently, $B_{y}^{\prime}=\frac{h}{h \pm \delta} B_{y} \approx$ $\left(1 \mp \frac{\delta}{h}\right) B_{y}$.

The potential function is of the form

$$
\phi(y, z)=\frac{1}{2 \pi} \int_{-\infty}^{+\infty} \frac{d k}{k} \int_{-\infty}^{+\infty} d \zeta N_{S}(\zeta) \frac{\cosh k\left(\frac{1}{2} d+y\right)}{\sinh k d} \cos k(\zeta-z) ;
$$

after some mathematical manipulations, the details of which are reported in the Appendix B of Ref. 5, we obtain for $-\frac{1}{2} h<z<\frac{1}{2} h$

$$
b_{y}(0, z)=-\frac{N_{S}}{2}+\frac{N_{S}}{2 \pi}[\arctan X+\arctan Y]
$$




$$
b_{z}(0, z)=-\frac{N_{S}}{\pi}\left[\ln 2-\ln \sqrt{1+X^{2}}-\ln \sqrt{1+Y^{2}}\right]
$$

and away from the pole $|z|>\frac{1}{2} h$,

$$
\begin{aligned}
& b_{y}(0, z)=\frac{N_{S}}{\pi}[\arctan T-\arctan S] \\
& b_{z}(0, z)=\frac{N_{S}}{\pi}\left[\ln \sqrt{1+S^{2}}-\ln \sqrt{1+T^{2}}\right]
\end{aligned}
$$

$X, Y, S$ and $T$ were defined in the previous subsection. The above results show that the field error $b_{y}(0, z)$ just below the pole has a value less than $N_{S}$ due to the influence of the pole length error over the rest of the wiggler and also that as we move away from the pole the perturbation field decreases exponentially.

\section{Displacement error}

This is a systematic error that arises from alignment tolerances in the assembly of a wiggler. If the two jaws of the undulator are displaced by a distance $\delta$, see Fig. 1c), the total magnetic field in the gap region is the sum of the contributions of each jaw, i.e. $B=B^{(+)}+B^{(-)}$. Neglecting the harmonics components and after some simple algebra (see Ref. 5) we write,

$$
B_{y}(0, z) \approx B_{y}^{\text {perf }}(0, z) \cos \frac{\pi}{l} \frac{1}{2} \delta \quad \text { and } \quad B_{z}(0, z) \approx B_{y}^{\text {perf }}(0, z) \tanh \frac{\pi}{l} \frac{1}{2} d \sin \frac{\pi}{l} \frac{1}{2} \delta
$$

where $B_{y}^{\text {perf }}$ denotes the field in a perfect wiggler.

The simulation results indicate that this systematic error is relatively less important than errors type $a$ and $b$. It has also been pointed out [9] that the relative displacement of the upper and lower jaws can be used to tune the laser frequency with significant advantages, rather than using the usual approach of closing the gap. 


\section{Numerical Analysis}

The undulator errors which arise from the unavoidable machining errors of both yokes tend to reduce the gain of an FEL with respect to the performance of one with an ideal wiggler. The impact of the errors will depend on the strength of the error at each pole, its spatial structure underneath the pole, and the statistical correlation between field errors at different poles [9]. The results presented in the previous section provide a systematic and unambiguous procedure to evaluate the degradation of the ATF-FEL performance: We have developed a simple code to follow a single electron in the undulator feld with a single gap or pole-thickness error, as well as a random distribution of them along the 60 cm length of the undulator. The amplitude of the error at each pole $b\left(z_{\text {pole }}\right)$ is given by an uncorrelated gaussian random variable, i.e.,

$$
<b\left(z_{\text {pole }}\right)>=0 \quad, \quad<b\left(z_{\text {pole }}\right) b\left(z_{\text {pole }}^{\prime}\right)>=\Delta^{2} \delta\left(z_{\text {pole }}-z_{\text {pole }}^{\prime}\right)
$$

where the angular brackets denote average over an ensemble of wigglers and $\Delta^{2}$ is the mean-squared deviation from the perfect wiggler gap or pole thickness dimensions. The equivalent magnetic field errors are expressed as $b_{y}(0, z)=b\left(z_{\text {pole }}\right) F(z)$ where $b\left(z_{\text {pole }}\right)$ is an stochastic variable satisfying Eq. (9) and $F(z)$ has the functional form shown above for gap and pole length errors.

Since as we pointed out in the previous section the error fields show an exponential fall-off, we only keep the influence of near-neighbors in the numerical computation. The error field $b_{y}$ of one of the realizations from the ensemble of statistically identical wigglers is shown in Fig. 4.

In Fig. 5 we show the standard deviation of the $x$-position at the end of the wiggler as a function of the its mechanical errors $\frac{\delta B}{B}$ in percents and for comparison we show also the results obtained with the Kincaid model given by $\left\langle\delta x^{2}>\approx \frac{K^{2} k_{w}^{2}}{\gamma^{2}}<\frac{\delta B^{2}}{B^{2}}>\frac{1}{2} \lambda_{w} \frac{z^{3}}{3}\right.$ with an $€ q u i v a l e n t$ random magnetic field distribution (i.e., $\Delta^{2} \equiv<\delta B^{2}>$ ). Likewise, in Fig. 6 we show the phase deviation at the end of the undulator plus or minus one mean-squared deviation $\sqrt{<\delta \zeta^{2}>}$ and compare it with the phase deviation $\left\langle\delta \zeta>\approx-\frac{K^{2} k_{w}^{3}}{\left(1+K^{2}\right)}<\frac{\delta B^{2}}{B^{2}}>\right.$ $\frac{\lambda_{w} z^{2}}{8}$ as given by the Kincaid model with an equivalent random magnetic field. 
To have negligible loss of gain we must have $\left\langle\delta x^{2}>\ll R_{L}^{2}\right.$ where $R_{L} \approx 220 \mu \mathrm{m}$ is the laser spot size and $\delta \zeta \ll \pi$. These constrains unable us to establish machining tolerances below $0.4 \%$ for the undulator; both limits are shown as horizontal lines in Fig. 5 and Fig. 6.

\section{Conclusions}

We have presented a mathematical model of errors in an iron-dominated wiggler; the perturbation fields created by gap and pole length errors have been explicitly derived. These fields show that $B_{y}(y, z)$ underneath the pole with the error is not $\frac{\Delta \Phi_{S}}{d}$ or $N_{S}$ but these values are reduced by the interaction of the error at $z_{\text {pole }}$ with the rest of the wiggler. These fields have been used to make a realistic modeling of a random distribution of gap and pole length errors. No ad-hoc assumptions regarding the field behavior underneath the pole or the functional form of the correlation function need to be made.

We have established constraints on the iron yoke machining tolerances needed to avoid degradation of the FEL gain due to random walk of the beam and phase deviations. The required precision of $0.4 \%$ can be readily obtained using the wire electric discharge method.

\section{References}

1. K. Batchelor et al., Status of the free-electron laser project at the Brookhaven Accelerator Test Facility, this conference;

K. Batchelor, I. Ben-Zvi, R. Fernow, J. Gallardo, H. Kirk, C. Pellegrini, A. van Steenbergen, Nucl. Instr. and Meth. A296 (1990) 239.

2. I. Ben-Zvi, Z. Jiang, G. Ingold, L..Yu, W. Sampson, Proc. of the Sag Harbor Workshop, J. Gallardo editor, BNL-42747 (1990).

3. I. Ben-Zvi et al., Brookhaven National Laboratory Report, BNL 52273, (1990), 65 A. Bhowmik, et al., Proc. of the 1990 International Conference on Laser'90, San Diego, unpublished.

4. I. Ben-Zvi et al., Performance of a superferric, high field subcentimeter undulator, this conference.

5. J. Gallardo, Brookhaven National Laboratory, BNL-45798, (1991). 
6. P. Morse and H. Feshbach, Methods of Theoretical Physics, pp. 710, pp. 797, McGrawHill Book Co., 1953;

S. Krinsky, "Wigglers for the National Synchrotron Light Source," Proceedings of the Wiggler Meeting, Frascati, June 29-30, 1978, A. Lucca, A. Reale and S. Stipcich, editors, December 1978; M.W. Poole, "Design and Technology of Undulators", Proceedings of CERN Accelerator School, Chester, April 1989, DL/SCI/P677A, Daresbury Laboratory.

7. K. Halbach, "Insertion Device Design", Lawrence Berkeley Laboratory Report V-88111.1-16 and "1990 Magnet Technology Lectures at LBL", ibid., V-902-2.1-6.

8. K. Halbach, "Summary of the 3-D Hybrid Theory with some Applications to the assessment of perturbation effects", L5BL Report LSBL-034, 1990; "Calculation of magnetic error fields in hybrid insertion devices", Nucl. Inst. Meth. A291 (1990) 408.

9. B. M. Kincaid, Nucl. Instrum. Meth. A291 (1990) 363 ; B. M. Kincaid, J. Opt. Soc. Am. B2 (1985) 1294 . For additional references see Ref. 5. 


\section{Figure Captions}

Figure 1: Schematics of the super-ferric micro-undulator presently under construction at the Accelerator Test Facility at Brookhaven National Laboratory. $\lambda=0.88 \mathrm{~cm}$ is the period; $d=0.44 \mathrm{~cm}$ is the gap; $h=0.22 \mathrm{~cm}$ is the length of the pole and $a=0.12 \mathrm{~cm}$. a) a single gap error in the upper jaw, $\delta$ is the localized perturbation of the gap. b) a single pole-thickness error, $\delta$ is the perturbation of the pole extent. c) Both jaws are displaced with respect to each other by a distance $\delta$.

Figure 2: Graphic interpretation of the treatment of a single gap error. The boundary condition for the perturbation term is $\phi\left( \pm \frac{1}{2} d, z\right)=\Delta \Phi_{S}$, for $-\frac{1}{2} h<z<\frac{1}{2} h$ and 0 otherwise.

Figure 3: Graphic interpretation of the treatment of a single pole thickness error. $-\frac{\partial}{\partial} \phi\left( \pm \frac{1}{2} d, z\right)=N_{S}$, is the boundary condition for the perturbation term for $-\frac{1}{2} h<z<\frac{1}{2} h$ and 0 otherwise.

Figure 4: A typical error field with $\frac{\delta B}{B}=0.5 \%$. Only near-neighbors have been kept in the calculation.

Figure 5: Standard deviation of $x$-positions at the end of a $60 \mathrm{~cm}$ wigler versus the mechanical error in percent. For comparison, results of the Kincaid model are shown with a dashed-line. The horizontal straight line correspond to $\sqrt{\left\langle\delta x^{2}\right\rangle}=R_{L}=220 \mu m$. 
Figure 6: Phase deviation $\delta \zeta \pm \sigma$ the standard deviation at the end of the undulator versus the mechanical error in percent. Also, the Kincaid model prediction is shown with a dot-dashed line. The horizontal line corresponds to $\langle\delta \zeta>=\pi$. 


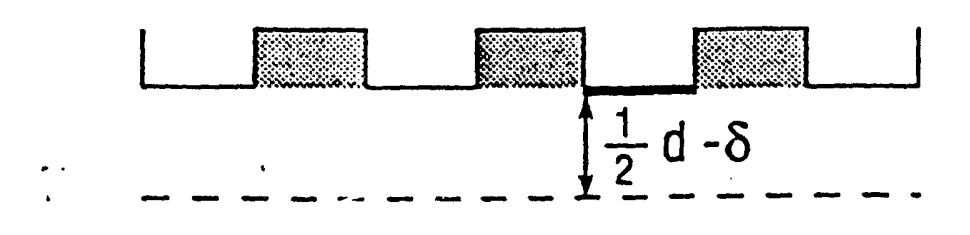

(a)
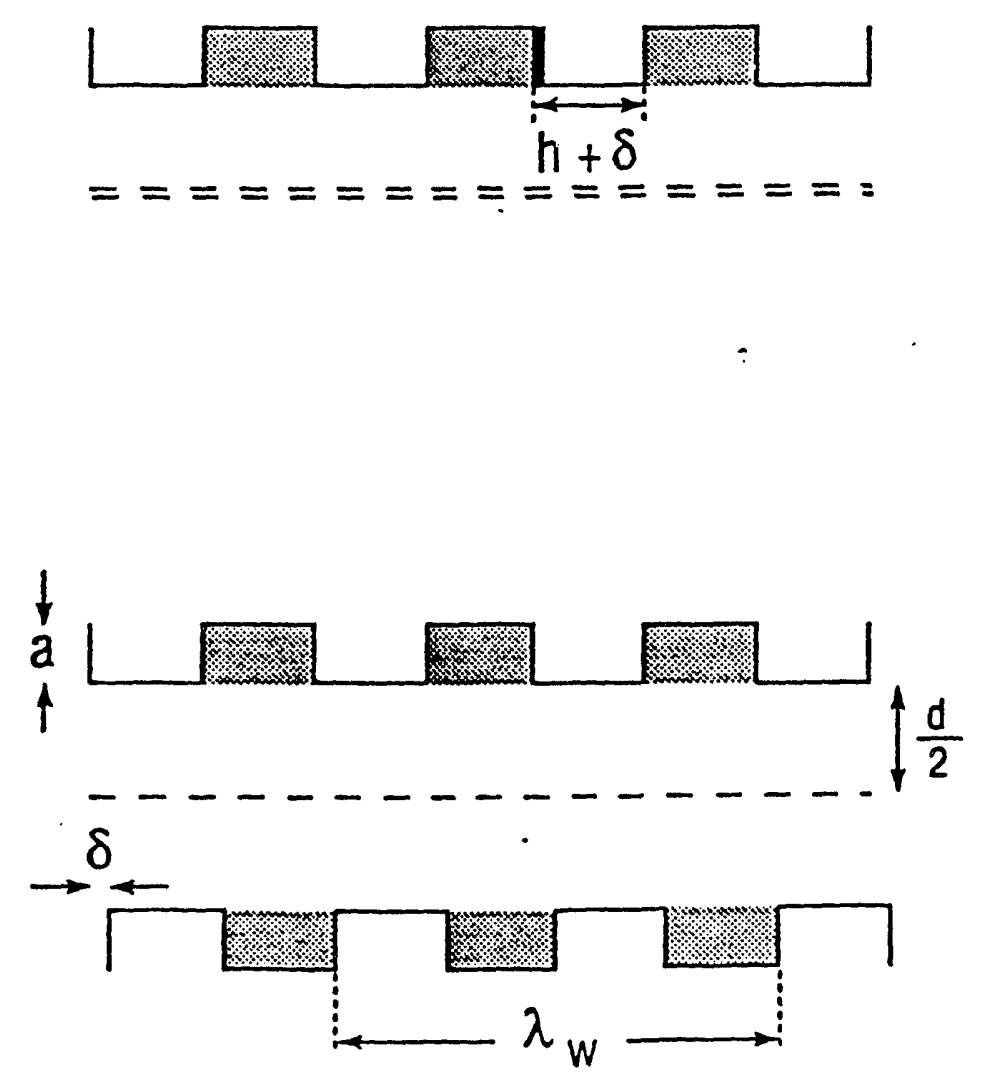

FIG. 1 

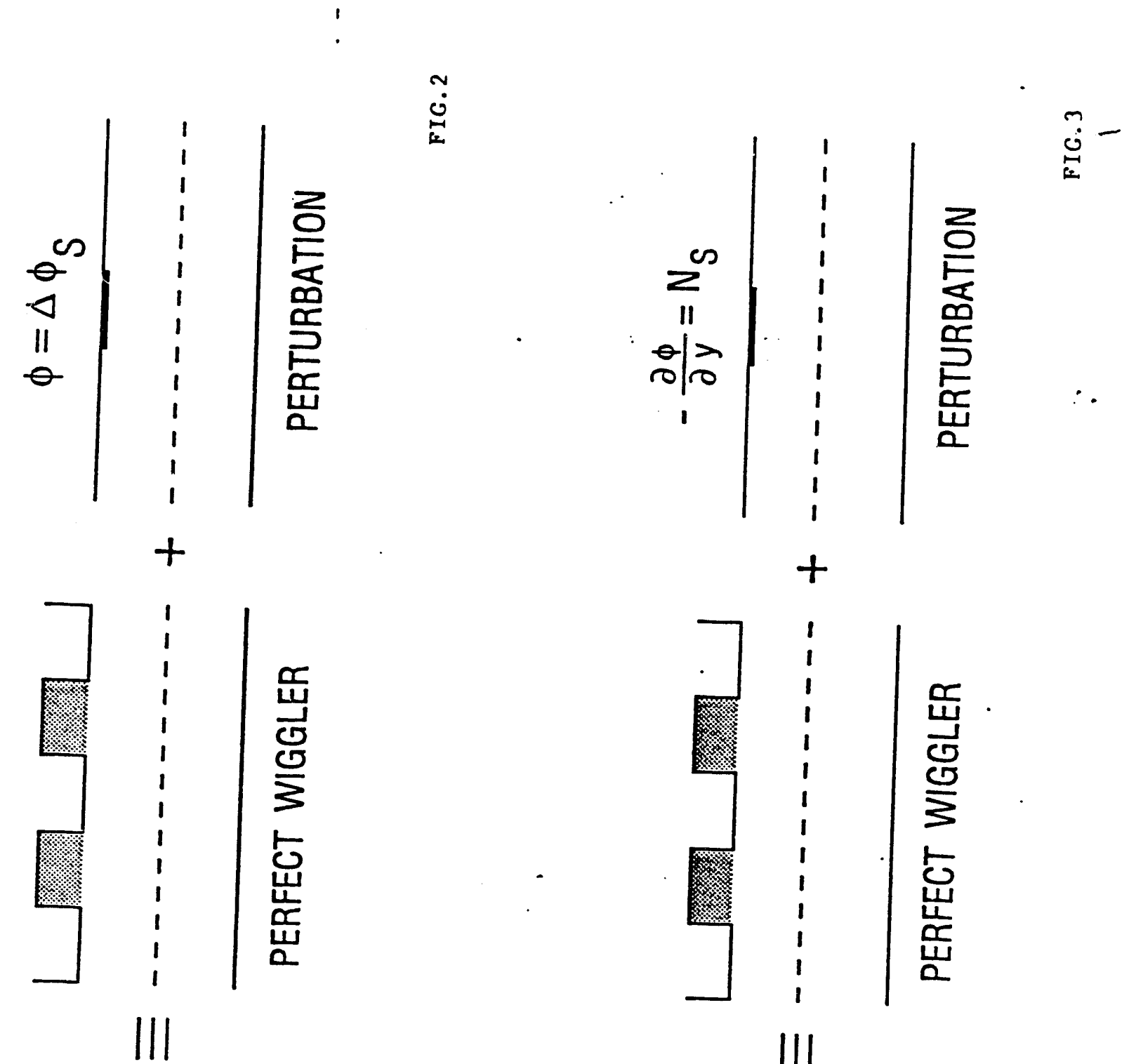

III
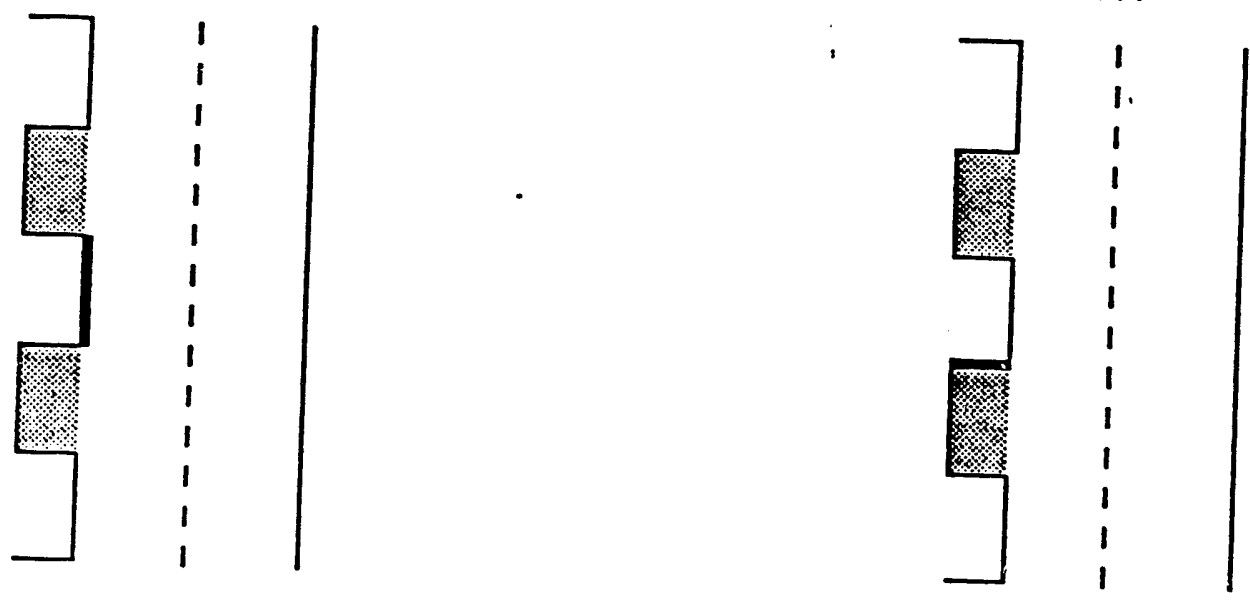


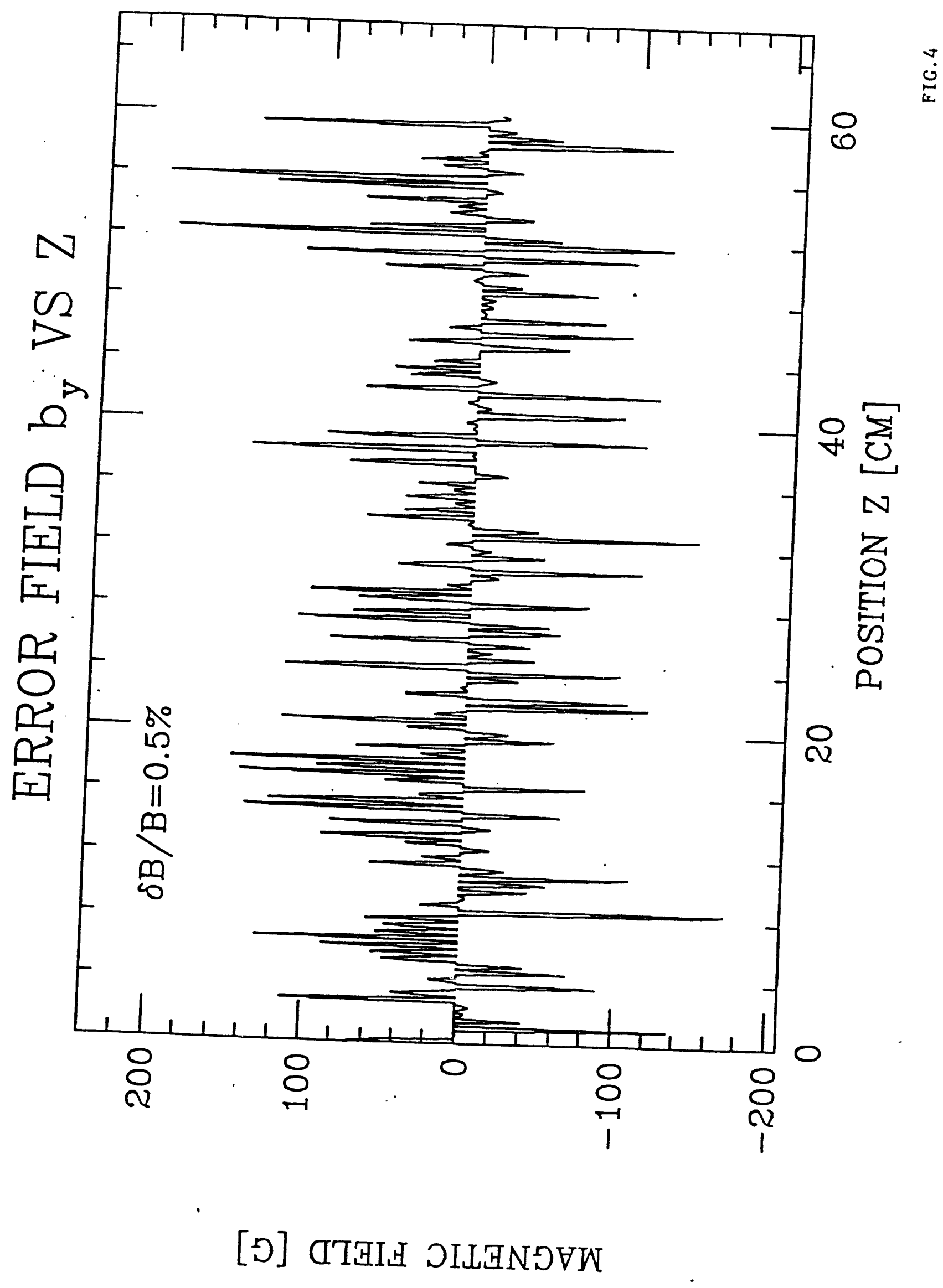




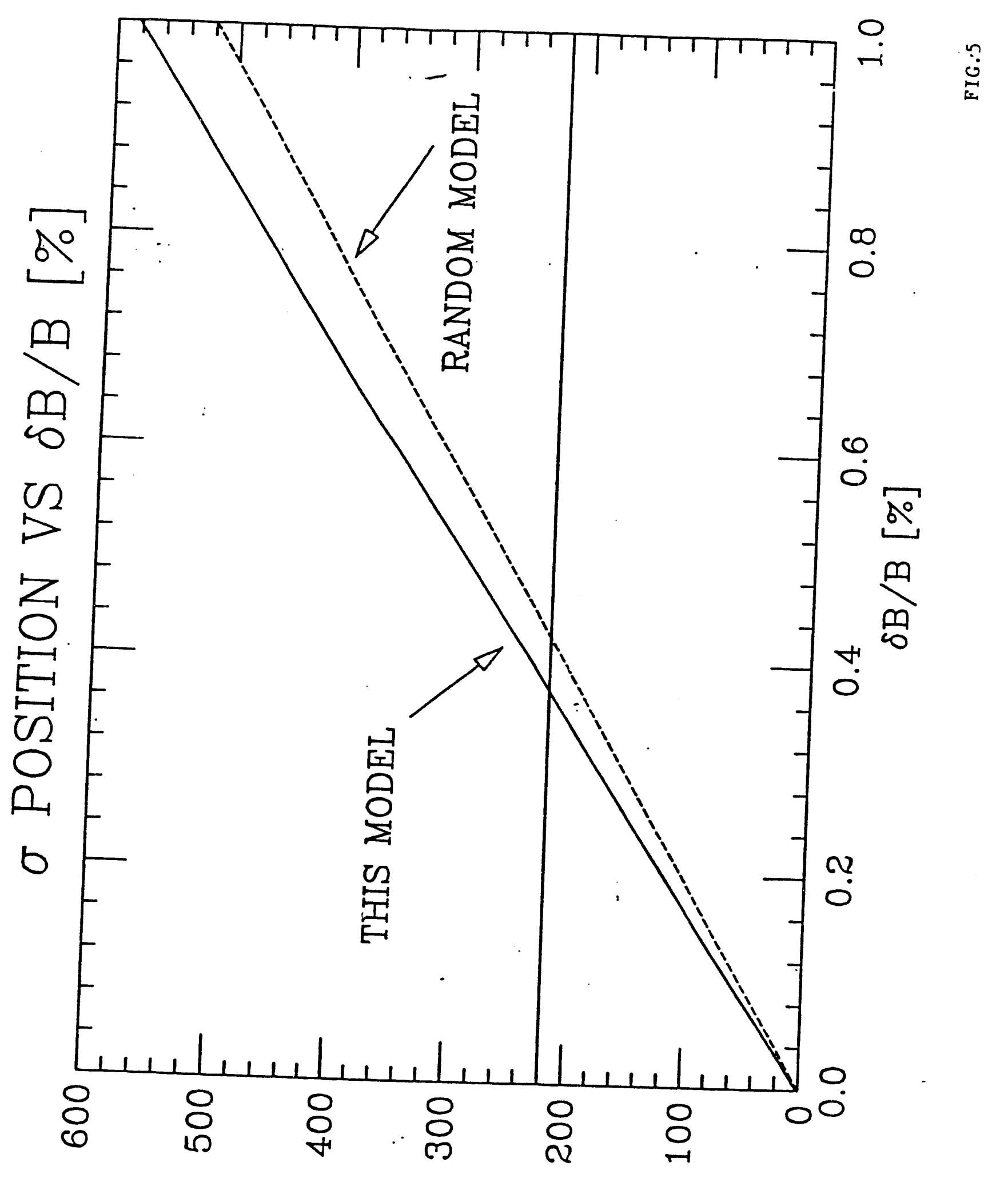

[urr] 


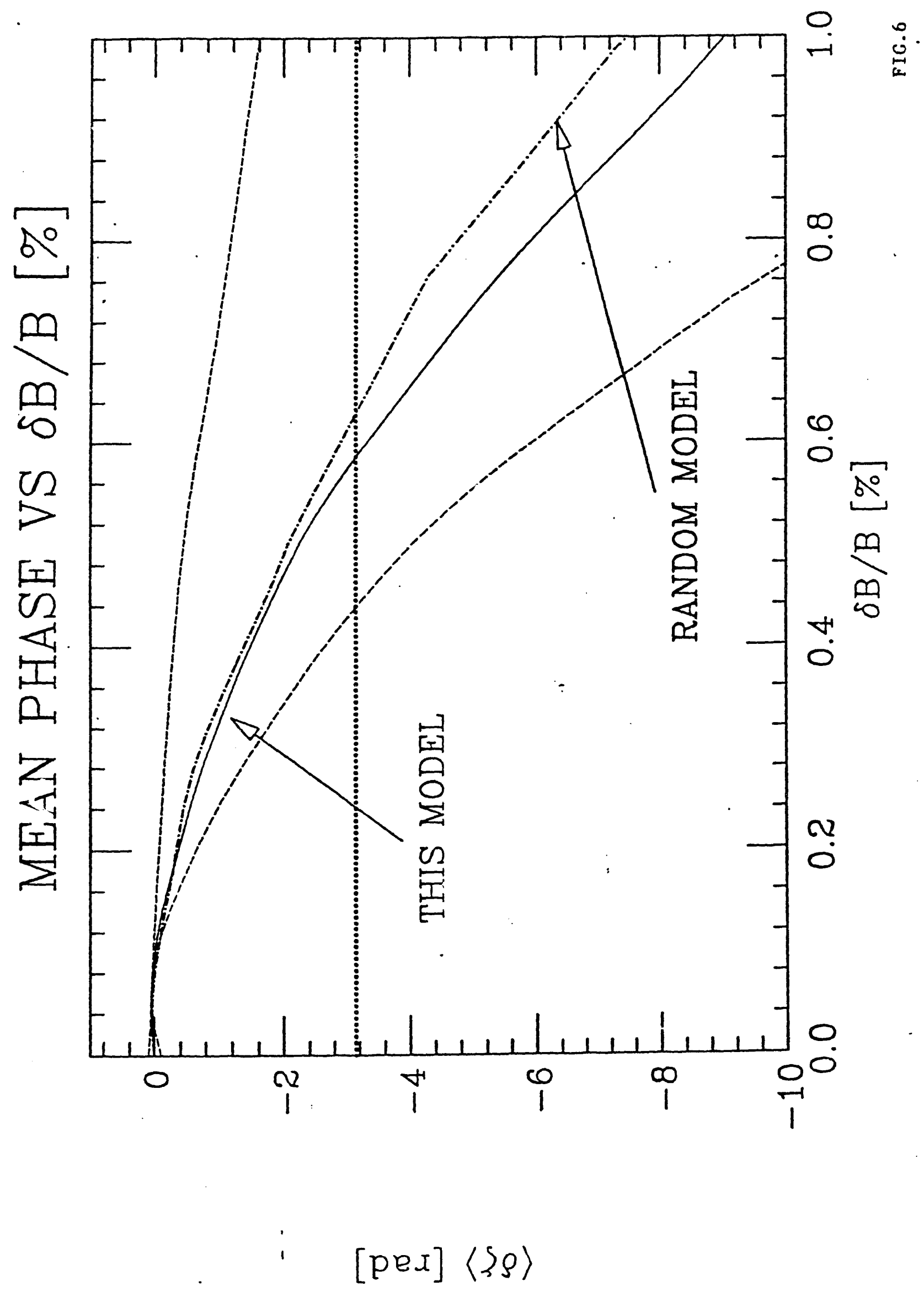



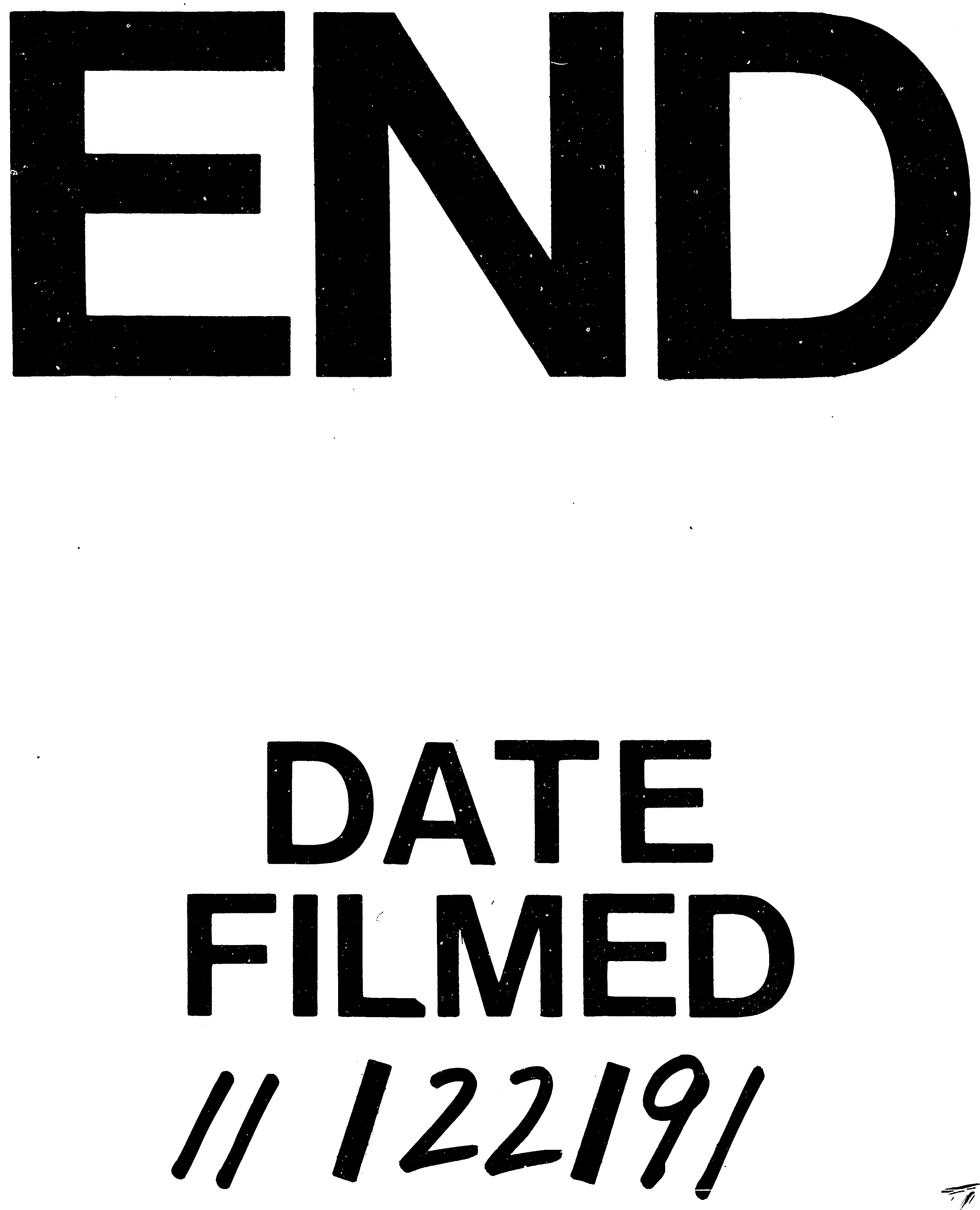
